\title{
Cultura institucional y su impacto en las prácticas de lectura y escritura en la universidad
}

\section{Institutional culture and its impact on reading and writing practices in the University}

Cultura institucional e seu impacto nas práticas universitárias de leitura e escrita

\author{
Elisa Bigi**, Marisol García-Romero ${ }^{\mathrm{b}}$, Edixon Chacón ${ }^{\mathrm{c}}$ \\ a* Doctora en Innovaciones Educativas, elisabigi05@gmail.com, ORCID 0000-0003-2556-5318, Universidad de Los Andes, Núcleo Táchira, Venezuela. \\ b Doctora en Filología Española, marisolgarcia1966@hotmail.com, ORCID 0000-0003-0970-1877, Universidad de Los Andes, Núcleo Táchira, Venezuela. \\ c Doctor en Psicología, edixonch@yahoo.com, ORCID 0000-0003-1519-5456, Universidad de Los Andes, Núcleo Táchira, Venezuela.
}

Forma de citar: Bigi, E., García, M. \& Chacón, E. (2018). Cultura institucional y su impacto en las prácticas de lectura y escritura en la universidad. Perspectivas, 3(2), 46-59.

Recibido: Enero 26 de 2018

Aceptado: Junio 01 de 2018

\section{Palabras clave \\ Alfabetización académica \\ Concepciones de los docentes \\ Cultura institucional \\ Perfil del egresado \\ Políticas lingüísticas}

Resumen: Este artículo constituye un segmento de la investigación sobre las concepciones de los profesores en torno a la producción de textos escritos en el ámbito universitario, desarrollada en la carrera de Educación, mención Básica Integral, de la Universidad de Los Andes, Núcleo Táchira. Se presenta una sección de los resultados referida al impacto de la cultura institucional en las prácticas de lectura y escritura. Nos apoyamos en la fundamentación teórica aportada por la alfabetización académica, las concepciones de los docentes, las políticas lingüísticas universitarias, y la noción de cultura institucional vista como el conjunto de prácticas, valores, convenciones, conocimientos, símbolos, lenguajes, tradiciones y creencias enraizados en las instituciones, trasmitidas por el monitoreo grupal, a través de la aprobación o desaprobación social. El estudio se hizo bajo el paradigma cualitativo, con enfoque interpretativo, y nos sustentamos en la teoría fundamentada, específicamente en la estrategia de la codificación teórica, para analizar la información obtenida a través de entrevis $\neg$ tas en profundidad efectuadas a diez docentes de la citada carrera. Los resultados surgidos muestran algunos elementos que conforman las prácticas institucionales respecto a la lectura y escritura en la carrera: cuáles profesores deberían ser responsables de enseñar la

\footnotetext{
*Autor para correspondencia

Elisa Bigi

elisabigi05@gmail.com
}

https://doi.org/10.22463/25909215.1588 


\section{Keywords}

Academic literacy

Teachers' conceptions

Institutional culture

Education graduated

profile

Language policies

\section{Palavras chave}

Alfabetização

acadêmica

Concepções de

professores

Cultura institucional

Perfil profissiográfico

Políticas linguísticas lengua escrita, carencia de acompañamiento institucional para orientar, motivar y publicar las producciones textuales de los profesores, usos inadecuados de la escritura por parte de los estudiantes (por ejemplo, plagio textual), y ausencia de políticas lingüísticas. Por otra parte, se advierte la necesidad de que en la universidad se expliciten políticas que orienten la alfabetización académica, las cuales deben incluir entre otros aspectos - la formación de los docentes y el compromiso de los distintos componentes institucionales para que se alcance este propósito.

Abstract: This paper constitutes a segment of the research about the production of written texts in the university field, developed at the University of Los Andes, Núcleo Táchira. It is presented a section of the results linked to Institute culture and its impact on reading and writing practices in the University. We rely on the theoretical foundation provided by the academic literacy theory, the conceptions of teachers, the language policies at the universities, and the notion of institutional culture seen as the set of practices, values, conventions, knowledge, symbols, languages, traditions and beliefs rooted in institutions and transmitted by means of group monitoring, through social approval or disapproval. The qualitative paradigm was assumed under the interpretive approach, and the method used was the Grounded Theory to analyze the information obtained through in-depth interviews with ten teachers of the aforementioned career. The results that emerged show some elements that make up the institutional practices with regard to reading and writing in the Education career: which teachers should be responsible for teaching written language, lack of institutional support to guide, motivate and publish textual productions of teachers, inappropriate uses of writing by students (for example, textual plagiarism), and absence of language policies. It concludes on the need for the university to specify policies that guide academic literacy, which must include - among other aspects - the training of teachers and the commitment of the different institutional components to achieve this purpose.

Resumo: Este artigo constitui um segmento da pesquisa respeito às concepções de professores sobre a produção de textos escritos no ambiente universitário, desenvolvida no programa de graduação em Educação Básica Integral (Licenciatura em Ensino Fundamental), da Universidade dos Andes, Núcleo Táchira. Apresenta-se uma seção dos resultados referentes ao impacto da cultura institucional nas práticas de leitura e escrita. Baseado na fundamentação teórica proporcionada pela alfabetização acadêmica, as concepções de professores, políticas linguísticas universitárias e a noção de cultura institucional vista como 
o conjunto de práticas, valores, convenções, conhecimentos, símbolos, linguagens, tradições e crenças arraigadas em instituições, transmitidas por monitoramento de grupo, através da aprovação ou desaprovação social. O estudo foi conduzido sob o paradigma qualitativo, com uma abordagem interpretativa, baseada na estratégia de codificação teórica, para analisar as informações obtidas por meio de entrevistas em profundidade com dez professores do referido programa de graduação. Os resultados mostram alguns elementos que compõem as práticas institucionais de leitura e escrita na carreira acadêmica: quais professores devem ser responsáveis pelo ensino da língua escrita, falta de apoio institucional para orientar, motivar e publicar as produções textuais de professores, usos da escrita inadequada pelos alunos (por exemplo, plágio textual) e ausência de políticas linguísticas. Além disso, é claro que há necessidade na universidade de políticas para orientar a alfabetização acadêmica, que deve incluir, entre outros aspectos, a formação de professores e o comprometimento dos diferentes componentes institucionais para alcançar esse intuito.

\section{Introducción}

La lectura y la escritura, aparte de ser procesos cognitivos, son también fenómenos históricoculturales, y siendo entonces procesos situados (Castelló, 2002) generan ciertos usos y prácticas con determinadas características, según los propósitos comunicativos y las particularidades de la comunidad donde se producen y manifiestan. Por ello, el estudio en torno a las prácticas de lectura y escritura en la universidad es un tema que mantiene su vigencia desde muchas de sus perspectivas. El solo hecho de que por medio de la lengua escrita se difunda, se construya, se profundice en el conocimiento científico como también en el requerido para el ejercicio práctico de las diversas profesiones constituye suficiente motivo para su análisis permanente. Como profesores de la carrera de Educación mención Básica Integral, de la Universidad de Los Andes, Núcleo Universitario Dr. Pedro Rincón Gutiérrez (ULA Núcleo Táchira), a diario advertimos tanto el quehacer de los docentes, como el desempeño de los estudiantes, y nos inquietan las preocupaciones e insatisfacciones permanentes respecto a las actividades que ambos actores educativos llevan a cabo en el campo de la lengua escrita: unos como enseñantes y los otros como aprendices, pues pareciera que pocas veces se tiene conciencia de que el alumno está entrando en una nueva cultura y que, como tal, requiere del acompañamiento de los expertos.

Sustentándonos en distintas reflexiones, experiencias didácticas e investigaciones que sobre el tema se han efectuado tanto en el ámbito nacional (Martins, 2012; Mostacero, 2012; Serrano, Duque y Madrid, 2012; Villamizar-Acevedo, Lozano-León \& Sierra-Garavito, 2017, por mencionar algunos) como internacional (Carlino, 2004, 2011, 2013; González y Salazar, 2015; Rincón y Gil, 2011; ArénizArevalo, 2017; entre otros) decidimos emprender un estudio cuyo propósito esencial fue determinar un conjunto de aportes teóricos y metodológicos que orientara la producción de textos escritos de índole académico y profesional, con este fin analizaríamos el quehacer con la lengua escrita a partir de las voces de profesores y estudiantes y podríamos ofrecer aportes que ayudasen a solventar las dificultades. Cabe mencionar que algunos segmentos de esta investigación se han publicado en artículos previos (Bigi, Chacón y García, 2013, 2014, 2017, 2018). 
Uno de los objetivos que nos planteamos en dicha investigación fue percibir las concepciones de los profesores en relación con la producción de los textos en el contexto de la carrera de Educación mención Básica Integral. En este artículo presentamos una de las subcategorías que emergieron durante el proceso de análisis de las entrevistas realizadas: la cultura institucional y su impacto en las prácticas de lectura y escritura, asunto que consideramos de interés por cuanto no conocemos investigaciones específicas sobre este tema en Venezuela y las realizadas en la ULA (cf. Andrade y García, 2015; García Romero, 2017; Guerrero y Zambrano, 2012; y Zambrano y Guerrero, 2015) han revelado que la tendencia de las investigaciones se centran en los enfoques de la enseñanza de la lengua escrita (gramatical y procesual), mas no en aspectos socioculturales.

\section{Metodología}

\section{¿Cómo entendemos la cultura universitaria?}

Carlino (2004) ofrece la siguiente explicación:

La cultura universitaria en un ámbito dado está configurada por las representaciones y las prácticas institucionales que con cierta continuidad temporal se vienen llevando a cabo en su seno:

Aquellos saberes y haceres propios de una determinada comunidad universitaria, caracterizados por su naturaleza social; su particularidad vinculada a ciertos grupos, lugares y tiempos específicos; su urdimbre que entrama conocimientos, creencias, actitudes, usos del lenguaje, herramientas, rasgos de afiliación o pertenencia e identidades sociales; tanto como modos de hacer consuetudinarios (p.1).

Esta misma investigadora agrega que este entramado de pensamiento y acción compartidos no suele ser cuestionado al interior de la comunidad para la cual resulta de alguna manera funcional, en la medida de que dicha trama otorga un carácter pseudo-natural y necesario. Por otra parte, es conveniente destacar que en una universidad se puede apreciar una cultura predominante, pero coexiste con un grupo de subculturas, las cuales muchas veces surgen de las idiosincrasias de las carreras.

Aplicando las ideas de Carlino (2004), expuestas en líneas anteriores, en el contexto de la presente investigación, se estaría hablando de una subcultura institucional, ya que se ubicaron algunas de las prácticas en la carrera de Educación, mención Básica Integral, de la ULA Núcleo Táchira, específicamente en lo relacionado con la lengua escrita, asunto situado en el campo de la cultura académica (Chacón-Guerrero et al., 2017; Gamboa-Suárez, 2016), dado que es una práctica establecida de esta cultura que hace posible ingresar y permanecer en ella.

\section{Concepciones de los docentes}

De acuerdo con Giordan y De Vecchi (1995), las concepciones constituyen un proceso personal a través del cual cada persona estructura su saber a medida que integra los conocimientos, en la mayoría de los casos durante un período bastante amplio de la vida. Ese saber se construye "a partir de su arqueología” (p. 92), en otras palabras, a través de la interacción con la cultura familiar, escolar, como también por influencia de los medios de comunicación y, luego, de la actividad social y profesional del adulto.

La definición de Moreno y Azcárate (2003) enlaza las ideas, creencias y conocimientos del profesor, pues plantea que las concepciones son “organizadores implícitos de los conceptos, de naturaleza esencialmente cognitiva y que incluyen creencias, significados, conceptos, proposiciones, reglas, imágenes mentales, preferencias, etc., que influyen en lo que se percibe y en los procesos de razonamiento que se realizan" (p. 267).

Otro de los sustentos teóricos que se tomó para este estudio es el de Pozo (2009), quien plantea que las concepciones son teorías implícitas. Este autor expone que:

(...) en muchos casos tal vez ni profesores ni alumnos podrían formular de modo explícito y riguroso sus creencias sobre qué es el conocimiento 
y cómo se adquiere. Pero eso no quiere decir que no asumen ciertos supuestos y creencias intuitivas, en lo esencial de carácter implícito, en su actividad diaria de aprender o enseñar (p. 71).

También señala que la mayor parte de los profesores universitarios no reciben una formación explícita sobre los procesos de aprendizaje y enseñanza; por tanto, su experiencia como docentes (y también como alumnos) les provee creencias sólidas sobre cómo aprenden sus alumnos, cómo se tiene que evaluar. Lo mismo ocurre con los estudiantes, quienes, por los años acumulados en el rol de alumnos, también han creado expectativas y creencias en relación a lo que se debe realizar en las situaciones de enseñanza y aprendizaje.

Según Morales (2000), las concepciones en torno a la lengua escrita de los docentes se construyen sobre la base de sus experiencias de vida, teniendo las siguientes fuentes de aprendizaje: en primer término, por las experiencias de aprendizajes familiares y escolares; segundo, por el desempeño como usuarios de la lengua escrita; tercero, cuenta la formación académica inicial y profesional de la docencia; cuarto, la cosmovisión o concepción del mundo; y quinto, por la formación profesional permanente (actualización docente). Todo esto corrobora que las concepciones no solo determinan la manera de ver la realidad, sino que guían y orientan la forma de actuar en el aula.

\section{Políticas lingüísticas institucionales universitarias}

Para definirlas se recurre al concepto operativo y provisorio de política universitaria de lectura y escritura formulado por la Red de Lectura y Escritura en la Educación Superior (REDLEES, 2013). Este organismo la concibe como una manera de comprender y de efectuar la formación en lectura y escritura que ha de incorporar -como mínimo-dos tipos de consideraciones: las primeras, de carácter explícito, sobre las relaciones entre la universidad y la sociedad, "y sobre el papel que las prácticas lectoras y escriturales deberían tener en el desempeño profesional y en la actuación de los egresados como personas en nuestras sociedades" (p. 26). Las segundas, serían consideraciones generales referidas al modo como esta formación debería proyectarse en los planes de estudio de la institución; en otras palabras, incluiría la didáctica más adecuada, la forma de organizar el currículum para el desarrollo de la lengua escrita, la formación del profesorado, entre otros elementos.

Sobre este último aspecto, señalaremos un segmento del Diseño Curricular de la carrera de Educación, mención Básica Integral (Universidad de Los Andes, 2009), el cual entre sus lineamientos establece el dominio de la lengua escrita en el perfil de egreso:

Se espera que el estudiante desarrolle, use y consolide sus capacidades de comunicación, es decir, las competencias de producción y de comprensión discursivas, entre ellas: fluidez oral, riqueza léxica, manejo consciente de la normativa y del registro formal, habilidades para la escritura (especialmente, el proceso de composición de textos académicos: artículos, ensayos, informes de investigación, monografías...), escucha activa, y lectura analítico-crítica (p. 88).

Con este señalamiento se puede observar que, en efecto, hay ciertos contenidos curriculares referentes a lo que se aspira del estudiante en el transcurso de su escolaridad, no obstante, hacen falta políticas que especifiquen de qué forma todas las instancias institucionales apoyarán el desarrollo de las competencias lingüísticas, bien de los profesores bien de los alumnos.

\section{Alfabetización académica en carreras de formación docente}

En las últimas décadas el surgimiento del concepto alfabetización académica (AA), en el contexto de la educación superior, ha sido esencial para fomentar un giro en las prácticas de lectura y escritura desarrolladas en este ámbito. Se la entiende como un proceso complejo: va más allá del aprendizaje de conocimientos lingüísticos específicos para adentrarse en las maneras como se 
emplea la lengua escrita en las distintas disciplinas, por lo que engloba los géneros académicos y profesionales específicos de las cátedras y de las carreras. Para alcanzar estos conocimientos, se requiere de la intervención pedagógica de todo el profesorado, no solo de los especialistas en lenguaje (Carlino, 2013).

Rojas y Jiménez (2012) expresan que, en una dinámica de influencia mutua, el contexto condiciona las prácticas de lenguaje que se realizan a través de la lectura y la escritura, al mismo tiempo que posibilitan y determinan las transformaciones en los contextos en los que se utilizan. Se asume que el desarrollo de la lengua escrita influye en el desarrollo de la lengua oral y, siendo ambas elementos culturales primordiales, la universidad ha de ocuparse de su orientación y acrecentamiento. Para tal fin, las instituciones tienen que explicitar sus políticas lingüísticas, en las cuales se determine la responsabilidad de las autoridades, los docentes y los mismos alumnos en este proceso.

\section{Perfil del egresado de educación primaria y competencias lingüísticas}

El perfil profesional en el currículo se presenta de dos maneras: en primer término, se generaliza, es decir, va dirigido a la carrera de Licenciatura en Educación, indistintamente de las menciones; $\mathrm{y}$, en segunda instancia, define las competencias profesionales del egresado en la mención Básica Integral. Al respecto, el perfil del egresado se fundamenta en la formación y consolidación de cinco grupos de competencias, a saber: a) básicas; b) de formación integral y ética; c) básicas profesionales; d) profesionales específicas; y e) de formación práctica e investigativa.

En este perfil se aprecia el eje transversal Desarrollo de la Lengua, lo que subraya la importancia del mismo dentro de esta carrera. Este diseño curricular destaca que todas las áreas de aprendizaje deben concurrir con este eje por medio de variadas experiencias; la práctica pedagógica es esencialmente una actividad intersubjetiva y dialógica en la que se da un uso consciente y adecuado del lenguaje, de acuerdo a los diversos propósitos del acto comunicativo. Es así que en la vida universitaria existe una producción continua de textos orales y escritos, tanto en las aulas como en la comunidad extraescolar.

\section{Ámbito metodológico}

La investigación fue de naturaleza cualitativa, dentro del paradigma interpretativo, dado que la intención era esencialmente comprender y describir la realidad en el contexto educativo por medio del análisis de las concepciones de quienes participan en las situaciones objeto del estudio (cfr. Latorre, Rincón y Arnal, 2005).

Para los lapsos académicos en que se aplicaron las entrevistas en profundidad en el Departamento de Básica Integral tenía adscritos 19 profesores, de los cuales participaron como sujetos de investigación diez (10) docentes de las distintas áreas académicas de la carrera de Educación, mención Básica Integral. Siguiendo la línea del muestreo teórico, (Strauss y Corbin, 2002), la selección de los profesores para las entrevistas se hizo con base en estos criterios: tener un mínimo de dos años como docente en la carrera; se dio prioridad a quienes pertenecieran a las áreas vinculadas con la enseñanza de la lengua, sin embargo, se logró ubicar a un profesor por cada área académica que conforman el departamento que administra la carrera; tener disposición de tiempo y actitud cordial para conversar sobre el tema objeto de estudio. La tabla 1 muestra la distribución de los diez profesores entrevistados dentro del contexto de la carrera. Como se puede observar solo tres de los diez profesores participantes se relacionan directamente con la enseñanza de la lengua 
Tabla 1. Distribución de los profesores entrevistados dentro de las áreas académicas de

la carrera de Educación, mención Básica Integral ${ }^{1}$

\begin{tabular}{|c|c|c|c|c|c|}
\hline Departamento & $\begin{array}{c}\mathrm{N} .^{0} \mathrm{de} \\
\text { profesores } \\
\text { entrevistados }\end{array}$ & Áreas académicas & $\begin{array}{c}\text { Grado } \\
\text { académico }\end{array}$ & $\begin{array}{c}\text { Condición } \\
\text { laboral }\end{array}$ & $\begin{array}{c}\mathrm{N}^{\circ} \\
\text { asignado en } \\
\text { el } \\
\text { Documento } \\
\text { primario }\end{array}$ \\
\hline \multirow{6}{*}{ Básica Integral } & \multirow{6}{*}{6} & Lectura y Escritura & Doctor & Ordinario & 1 \\
\hline & & Currículo & Magíster & Ordinario & 8 \\
\hline & & Extensión Universitaria & Magíster & Ordinario & 3 \\
\hline & & Ciencia Integrada & Magíster & Ordinario & 5 \\
\hline & & $\begin{array}{c}\text { Formación para el } \\
\text { Trabajo y Expresión } \\
\text { Artística }\end{array}$ & Magíster & Ordinario & 6 \\
\hline & & Salud y Recreación & Magíster & Ordinario & 9 \\
\hline $\begin{array}{l}\text { Español y } \\
\text { Literatura }\end{array}$ & 2 & $\begin{array}{l}\text { Enseñanza y Práctica } \\
\text { del Español }\end{array}$ & Especialista & Ordinario & 10 \\
\hline \multirow{3}{*}{ Pedagogía } & \multirow{3}{*}{2} & Literatura & Magíster & Contratado & 7 \\
\hline & & \multirow{2}{*}{ Práctica Profesional } & Doctor & Ordinario & 4 \\
\hline & & & Magíster & Contratado & 2 \\
\hline
\end{tabular}

Las entrevistas pretendían conocer los puntos de vista, las experiencias de los profesores brindando un ambiente natural de conversación entre iguales (Taylor y Bogdan, 1992) y no se sintiera la presión del intercambio formal y cerrado de preguntas y respuestas relacionados con asuntos académicos. Con este fin se elaboró un cuestionario abierto que orientó el diálogo con los profesores seleccionados. Cabe destacar que se realizó una prueba piloto del guion de la entrevista y su resultado fue totalmente satisfactorio. Algunas de las preguntas realizadas durante la entrevista vinculadas con el segmento de la investigación que presentamos en este artículo fueron: ¿usted piensa que a los estudiantes universitarios hay que enseñarlos a escribir?, ¿quiénes deberían ser los responsables de esta labor?, ¿qué se pudiera hacer institucionalmente?, ¿usted considera que el contexto cultural universitario influye en la producción escrita de los estudiantes? ${ }^{2}$

El método seleccionado fue la teoría fundamentada (Grounded Theory), la cual apoya la generación de teoría sustentada en los datos (Strauss y Corbin, 2002). El proceso de codificación teórica comienza con la codificación abierta, mediante la cual se busca descubrir conceptos en los datos a través de sus propiedades y dimensiones. Seguidamente en la codificación axial se describen las categorías principales que emergen de los datos y a partir de la codificación selectiva se busca dar sentido a los resultados mediante su conexión con las teorías que los respaldan. Como producto de codificación abierta y axial se derivó en un sistema de categorías emergentes (de la que se tomó una sección para esta presentación). La información obtenida se procesó con el software estadístico ATLAS-ti, versión 7.5, por sus ventajas para archivar las 
entrevistas, segmentar los textos en citas, realizar la codificación, representar el trabajo conceptual en redes, entre otras.

\section{Resultados y discusiones}

\section{Las prácticas institucionales}

En la figura 1 se reflejan los componentes de la subcategoría que analizaremos en este artículo: Cultura institucional, elemento que se registró dentro de la categoría Caracterización del contexto y emergió de la dimensión Prácticas institucionales.

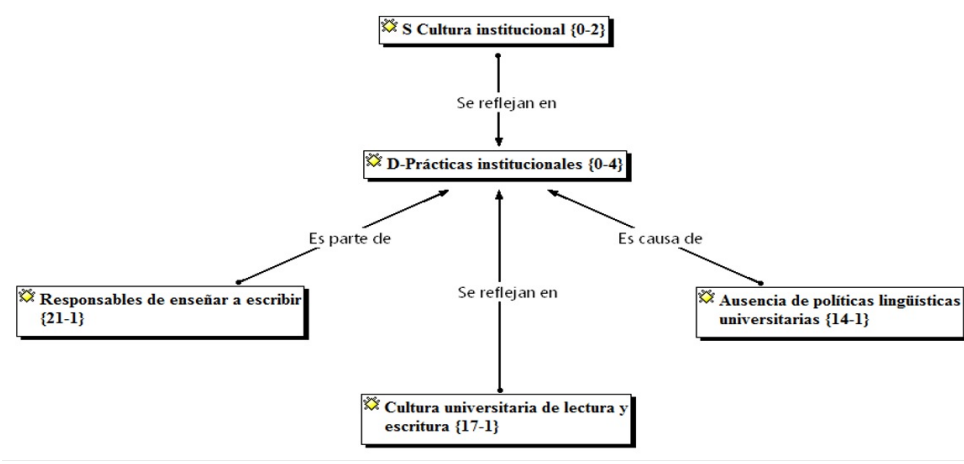

Figura 1. Subcategoría Cultura institucional

Los centros educativos se constituyen gracias ala interacción de distintos factores internos y externos; por eso, tienen formas de actuación, costumbres, creencias, etc., que las distinguen unas de otras. Entre esas formas de actuación se encuentran las Prácticas institucionales, relacionadas con la lectura y escritura. En las entrevistas afloró esta dimensión a través de tres elementos: Responsables de enseñar a escribir, Cultura universitaria de lectura y escritura y Ausencia de políticas lingüísticas universitarias.

El elemento más referenciado fue Responsables de enseñar a escribir, todos los entrevistados afirmaron que en la universidad se debe continuar la enseñanza de la lengua escrita; por ejemplo, uno de los entrevistados manifestó lo siguiente:

La gente de matemática se queja de que los estudiantes no saben leer ni interpretar, ni escribir ni resolver problemas, pero la gente de matemática -cuando digo la gente me refiero a los profesores de matemática- deberían guiar a los estudiantes en cómo leer, interpretar y redactar los problemas. $[1: 37][147: 151]^{3}$

En este comentario, el informante expresa que los docentes de algunas áreas disciplinares señalan las dificultades de los estudiantes para elaborar las tareas de lectura y escritura propuestas durante las clases. Frente a esto, resalta que a estas disciplinas les incumbe orientar en torno a la manera en que se pueden abordar los textos propios de su ámbito, tanto para leer como para redactar.

Una acotación adicional agrega que los profesores de las cátedras vinculadas con el lenguaje, no son los únicos encargados de desarrollar estas competencias comunicativas: "Todos los profesores debemos encargarnos. Por lo menos señalar cuando observamos muchas debilidades, eso no es solo responsabilidad de los profesores de Lengua, de Español, de Lenguaje, de Comunicación, de Lectoescritura, sino de todos". [3:22] [88:91] En esta opinión coincidieron todos los entrevistados lo cual consideramos relevante por cuanto solo siete de ellos tienen a su cargo asignaturas del área de lengua materna.

Aun cuando se presentó esta coincidencia en la respuesta de los profesores también de los testimonios se pudo inferir que, en algunas unidades curriculares, hay carencia de conocimientos por parte del docente en torno a cómo guiar al alumno en las actividades lectoras y de producción textual necesarias para construir su aprendizaje, tal como se percibe en este testimonio:

(...) cómo se explica que uno le pida a un estudiante un ensayo $\mathrm{y}$, entonces, cada estudiante tiene una idea distinta de lo que es el ensayo y yo también creo que yo pienso distinto a ellos, entonces digo que a lo mejor soy yo. Por eso me da la impresión de que los profesores no sabemos escribir o que no sabemos revisar los trabajos que pedimos. [2:184] [180:185]

Sin embargo, algunos de los docentes advierten que sus mediaciones en el proceso de escritura serían de gran provecho para los alumnos, como se lee en este testimonio: "Entonces, yo digo, si los profesores de investigación, de matemática, de

${ }^{3}$ Estos números indican lo siguiente: en el primer corchete, el primer dígito identifica al documento primario (entrevista) y se corresponde con el orden asignado al entrevistado; mientras que la siguiente cifra hace referencia al número de cita en ese documento. Los números del segundo corchete precisan las líneas donde comienza y termina cada una de las citas. 
todas las materias se dedicaran a que el estudiante de verdad escribiera, pues escribiría, escribiría mejor". [2:82] [208:210]

Con respecto a la Cultura universitaria de lectura y escritura, este elemento se manifestó en seis de los entrevistados. Uno de los docentes dio este testimonio:

Y muchos entramos a la universidad y hasta que no tenemos un tutor que nos ayude, o un grupo de trabajo o una comunidad en el departamento, o un área que incentive, no publicamos. La cultura interna de la institución no fomenta la escritura. [1:174] [716:719].

El informante expone sobre una situación por la que atraviesa la mayoría de los profesores cuando ingresa a la universidad, en cuanto a la producción intelectual. Destaca la ausencia de un acompañamiento institucional para orientar, motivar y publicar los textos que los docentes producen; cada quien se abre paso bien por la intervención de un colega experto o bien por algún grupo que apoye este tipo de trabajos. Enfatiza que en este nivel educativo no se estimula la práctica escritural.

Otro comentario menciona las debilidades ortográficas de los estudiantes y las compara con los errores que aparecen en los avisos y en otros textos publicados en carteleras dentro de la universidad. Es decir, pareciera que en el ámbito universitario es común que en estas publicaciones aparezcan algunos errores y el informante sugiere que, ante esta circunstancia, los alumnos repiten esas faltas de lenguaje en sus escritos:

Los muchachos de hoy no saben ni escribir, a veces, su propio nombre y apellido; por ejemplo, el aspecto de la tildación es gravísimo. Y yo siempre les digo a ellos que la universidad no hace ejemplos de eso porque en las carteleras, en los avisos, en todo, nosotros vemos precisamente esos defectos (...). [10:64] [17:21]

A los segmentos anteriores, se suman las acentuadas referencias por parte de algunos entrevistados hacia el plagio de textos que acostumbran a hacer algunos alumnos:
Entonces lo que me consigo es que ellos, por ejemplo, buscan toda la información de Internet y copian y pegan, y no son capaces, a veces, ni de parafrasear o tomar algunas ideas de lo que han escrito algunos investigadores y a ponerse ellos a reflexionar sobre lo que leen. [3:28] [108:112]

El plagio de textos no es una práctica nueva en el recinto universitario. Aunque hay varias formas de copiar los textos, los entrevistados señalan que el fácil acceso a la información por Internet ayuda a esta indebida copia intelectual. Pero ese mismo acceso puede permitir a los profesores chequear si el trabajo que le entrega el alumno es una copia o es redacción propia.

El último elemento que emergió en esta dimensión fue Ausencia de políticas lingüísticas universitarias, asunto sugerido por cuatro de los entrevistados. Uno de los testimonios señala: "(...) yo pienso que en la universidad no hay una conciencia clara de cuál es la función de la escritura y que el que sabe escribir escribe lo que necesita y eso no es así". [1:38] [157:160]

Otro entrevistado comentó que en otras universidades había una dirección más clara sobre los textos que tenían que escribir los estudiantes, según la carrera que cursaban, y señaló: "Yo creo que a esta universidad le falta estructura, debería dar unos lineamientos, proporcionarle algunas herramientas al alumno, ofrecer la oportunidad de que el alumno escriba cada vez mejor y que se comprenda y haga comprender lo que escribe". [7:13] [68:71] A esta opinión se suma la siguiente: "Yo creo que en la universidad nuestra la escritura la han relegado por la forma como plantean los profesores sus asignaturas, sus clases, la metodología que emplean, se ha relegado". [9:8] [48:50]

Estos docentes piensan que se necesita establecer y divulgar lineamientos en torno a la escritura, para incrementar el nivel académico de este proceso en la comunidad universitaria. Resaltan que las carencias pedagógicas en las estrategias de escritura que los profesores aplican en sus clases inciden en la baja calidad de los textos producidos por los alumnos. 


\section{Reflexiones en torno a la cultura institucional}

La cultura institucional (también llamada cultura organizacional) se entiende como un conjunto de prácticas, valores, convenciones, conocimientos, símbolos, lenguajes, tradiciones y creencias enraizados en las instituciones que condicionan la actuación de los individuos. Este conjunto de elementos se transfiere de manera informal, es decir, representa la parte no escrita de la forma de ser institucional, no se impone a través de documentos escritos (manuales, reglamentos, políticas), sino que se transmite por medio de otros mecanismos como, por ejemplo, el monitoreo grupal, a través de la aprobación o desaprobación social (cfr. Daft, 2005; Toca y Carrillo, 2009).

Las instituciones educativas incluidas las universitarias también poseen sus propias culturas adscritas a programas de políticas públicas relacionadas con la gestión educativa (AguilarBarreto et al., 2017). Al respecto, Wesseler (2004) comenta que las universidades, las facultades, los departamentos e incluso las disciplinas científicas tienen sus particulares culturas, las cuales -en muchas ocasiones y en forma inconsciente-deciden sobre las acciones, las prácticas que en ellas se efectúan.

Lozano, Hernández, Ortíz y Chacón (2015) refieren que disertar de la escritura como cultura universitaria significa "hablar de la escritura como un actividad que caracteriza las prácticas académicas tanto de estudiantes como de profesores" (p. 155), en otras palabras, significaría hablar de una actividad 'hecha costumbre' en el mejor sentido de la palabra" (p. 155), pues se vincularía con prácticas académicas reflexivas y comprometidas con el desarrollo, producción y divulgación del conocimiento $\mathrm{y}$, por ende, con la transformación social. No obstante, estos mismos autores destacan que en los últimos años varios estudios reflejan un somero avance en el fortalecimiento de esta cultura, aun cuando se han efectuado grandes esfuerzos en varias instituciones universitarias.
Cuando en el segmento anterior se describieron las prácticas institucionales, se refirieron elementos como los responsables de enseñar a escribir en la universidad, la cultura universitaria de lectura y escritura y la ausencia de políticas lingüísticas universitarias. En estos, especialmente en los dos primeros, se destaca el papel que ha de desplegar el profesor como mediador entre los conocimientos y experiencias que traen los estudiantes y lo que van a encontrar al interior de unidades curriculares.

También es importante enfatizar en que existe la creencia de que el alumno debería llegar a la universidad con las competencias suficientes para desempeñarse convenientemente en las actividades lectoras y de producción textual requeridas en las cátedras; esto no es así, tal como lo explica Carlino (2011) al comentar el impacto que sufren los jóvenes que ingresan a la universidad y se topan con una cultura académica distinta a la del bachillerato:

El encuentro entre culturas indica que los modos de estudiar, leer y escribir son diferentes. Significa que en la escuela secundaria se ejercen cierto tipo de prácticas letradas y en la universidad, otras. En cada nivel existen expectativas bien distintas acerca de lo que deben hacer, pensar y valorar los alumnos. En la universidad, los docentes y la institución esperan que los alumnos lean, escriban, hablen, piensen y participen de ciertas maneras distintas a las esperadas en otros contextos" ("Ingresar y permanecer en la universidad pública", párr. 2).

De igual manera, se advierte, en comentarios de algunos profesores, el reconocer que todos ellos deben atender las deficiencias escriturales de los alumnos, pero también admiten no saber cómo hacerlo y, por ende, las dejan pasar.

También en la realización de los trabajos solicitados por los docentes, estos han subrayado la costumbre de ciertos alumnos por copiar textos sin identificar las fuentes. Hay estudiantes que presentan sus trabajos con estas características y no reconocen que hicieron un plagio, no se dan cuenta de lo incorrecto de su acción porque ha sido una práctica realizada durante gran parte de su vida estudiantil (que prolongan en la universidad), en la que rara 
vez se les ha señalado que eso constituye un delito intelectual; por tanto, es perentorio inculcarles la necesidad de especificar las fuentes bibliográficas o de otro tipo en sus trabajos.

Lomas y Ruiz (2002) aseveran que

(...) en la universidad, salvo honrosas excepciones, se actúa como si ese saber hacer se diera por hecho, aunque la realidad pronto evidencia un absoluto desconocimiento de las normas básicas de referencia bibliográfica y citación por parte del alumnado. Los estudiantes, acostumbrados a la copia, construyen "refritos" con la esperanza de no ser descubiertos al hacerlos pasar por originales ( $p$. 7).

En los últimos años el problema del plagio ha aumentado debido a las facilidades de copiar y pegar textos digitalizados a través de la Internet (cf. Caldevilla, 2010; Domínguez-Aroca, 2012; Martín, Hernández-Suárez \& Mendoza-Lizcano, 2017; Urbina, 2004). Además, en muchas ocasiones, no es posible reparar estos escritos; es costumbre que los trabajos sean entregas finales, lo que equivale a decir que no han pasado por revisión de borradores preliminares, reescrituras. De manera que muchos estudiantes no reciben una retroalimentación en torno a su composición textual.

Estas formas de proceder conducen a revisar la responsabilidad que deberían asumir los docentes como mediadores para ayudar a la incorporación de los alumnos en el lenguaje de cada una de las disciplinas, de forma que aprendan a buscar y plasmar ideas de diferente autoría, redactar textos que reflexionen respecto a esas ideas y, cuando corresponda, exponer sus puntos de vista particulares. Para lograrlo los profesores requieren de formación, dado que muchos de ellos -que no son del área de lengua materna- tienen carencias en la didáctica de la lectura y escritura, en su formación universitaria eso no estaba contemplado.

\section{Conclusiones}

Luego de presentar el análisis de la subcategoría Cultura institucional en el marco del estudio realizado, podemos concluir que -en el ámbito de la carrera de Educación, mención Básica Integral de la ULA Núcleo Táchira- los profesores reconocen su responsabilidad de enseñar a leer y a escribir en sus asignaturas y que necesitan apoyo institucional para esta labor. Se avista cierta preocupación entre ellos ante las carencias estudiantiles en lectura y escritura. Algunos afirman atender estos problemas, pero no son la mayoría, y también han señalado su déficit no solo en cuanto a conocimientos en didáctica de la lengua materna y la falta de tiempo (entre otros elementos) para intervenir en esta situación, sino también en lo relacionado a la estructura de los textos que solicitan a sus alumnos. Entonces, hay un contexto en el que se demandan tareas de lectura y de escritura, donde se dan instrucciones para su realización, pero la mayoría de las veces hay ausencia de acompañamiento en las mismas y cierta inseguridad en cuanto a cómo es el tipo de texto que se está requiriendo.

Esto es parte delas prácticas de lectura y escritura instaladas dentro de la carrera, prácticas que se repiten, en oportunidades se critican aisladamente, pero no ha habido una conciencia colectiva para cambiarlas. Por ende, es un escenario que amerita de transformación. Los profesores perciben la carencia de políticas lingüísticas institucionales para atender integralmente las necesidades y requerimientos del contexto. El establecimiento de este tipo de políticas es esencial para introducir la noción de AA en el seno de la ULA Núcleo Táchira, lo que complementaría la implementación del diseño curricular por competencias de las carreras de Educación (2009). Estas políticas deben incorporar principios teóricos educativos actuales, tales como la transversalidad y la interdisciplinariedad, ya que constituyen parte de la fundamentación del diseño curricular mencionado.

Bien afirma Ramírez (2017) que a la universidad le corresponde y es responsable de fomentar una cultura compartida en cuanto a los modos de enseñar la lectura y la escritura. Concretar estas políticas lingüísticas precisa de la labor conjunta de diversas instancias universitarias, académicas y 
administrativas: las primeras, se ocuparían de definir las políticas, sus objetivos, las acciones puntuales, los programas, entre otros, y las segundas, si se plantea la alfabetización académica universitaria como un hecho integral, entrarían a formar parte de este proceso dado que las transformaciones que se proyecten han de contar con el aval institucional.

\section{Referencias}

Aguilar-Barreto, A., Velandia-Riaño, Y. R., Aguilar-Barreto, C. P., \& Rincón-Álvarez, G. (2017). Gestión educativa: Tendencias de las políticas públicas educativas implementadas en Colombia. Revista Perspectivas, 2(2), 84-94.

Andrade, E. y García Romero, M. (2015). Investigaciones sobre escritura académica realizadas por la comunidad científica de la ULA (2009-2013): alcances y limitaciones. Educere, 19 (63), 465-468.

Aréniz-Arévalo, Y. (2017). Desarrollo de la comunicación oral y escrita como competencia genérica en la formación profesional de estudiantes de Ingeniería Civil. Revista Perspectivas, 2(2), 60-72.

Bigi, E.; García Romero, M. y Chacón, E. (2013). ¿Cuáles textos académicos leen los estudiantes universitarios en Educación?. Legenda, 17 (17), 127-145. Disponible en: http://erevistas.saber. ula.ve/index.php/legenda/article/view/4644

Bigi, E.; García Romero, M. y Chacón, E. (2014). Acercamiento al perfil del estudiante universitario según las concepciones docentes. Acción Pedagógica, 23, 30-37. Disponible en: http://www.saber.ula.ve/ bitstream/123456789/40897/1/dossier03.pdf

Bigi, E.; García Romero, M. y Chacón, E. (2017). Estrategias de enseñanza: análisis relacionado con la producción escrita en la universidad. Educ@ciónen Contexto,3(6),14-45.Disponible en: http://revistaeducacionencontexto. blogspot.com/p/publicacion-actual_22.html

Bigi, E.; García Romero, M. y Chacón, E. (2018). Una mirada a las concepciones docentes respecto a las tareas de escritura en la universidad. Akademos, 19 (1 y 2), 163-183. Disponible en: http://saber.ucv.ve/ojs/index. php/rev_ak/article/view/15839

Caldevilla, D. (2010). Internet como fuente de información para el alumnado universitario. Cuadernos de Documentación Multimedia, 21-157. Disponible en: http://revistas.ucm.es/ index.php/CDMU/article/view/21655

Carlino, P. (2004). Culturas académicas contrastantes en Australia, EEUU y Argentina: Representaciones y prácticas sobre la escritura y sobre la supervisión de tesis en el grado y postgrado universitarios. Trabajo presentado en la Reunión Internacional "Mente y Cultura: Cambios representacionales en el aprendizaje". Centro Regional Universitario Bariloche de la Universidad Nacional del Comahue. Disponible en: http://www.escrituraylectura.com.ar/ posgrado/articulos/Carlino_Culturas $\% 20$ academicas\%20contrastantes_04.pdf

Carlino, P. (2011). "Ingresar y permanecer en la universidad pública”. Entrevista publicada en el Suplemento de Educación del periódico El Eco de Tandil, 30 de abril de 2011, p. 5, en ocasión de celebrarse el IV Encuentro Nacional y I Latinoamericano sobre Ingreso a la Universidad Pública, Universidad Nacional de Centro de la Provincia de Buenos Aires (UNCPBA), 4-6 de mayo de 2011, Tandil, Provincia de Buenos Aires. Disponible en: https://media. utp.edu.co/referencias-bibliograficas/uploads/ referencias/ponencia/paula-carlino-ingresary-permanecer-en-la-universidad-2011pdfpcH4U-articulo.pdf

Carlino, P. (2013). Alfabetización académica diez años después. Revista Mexicana de Investigación Educativa, 18 (57), pp. 355381. Disponible en: http://www.redalyc.org/ articulo.oa? id=14025774003

Castelló, M. (2002). De la investigación sobre el proceso de composición a la enseñanza de la escritura. Signos, 35 (51-52), pp. 149162. Disponible en: https://scielo.conicyt.cl/ 
scielo.php?script $=$ sci_abstract\&pid=S071809342002005100011\&lng=es\&nrm=iso

Chacón-Guerrero, É., Eslava-Zapata, R., ChacónLobo, G., Gonzalez-Júnior, H. A., \& del Valle Buitrago-Rodríguez, M. (2017). Cooperación académica entre el sector productivo y las universidades: Una evaluación basada en las prácticas estudiantiles. Revista Perspectivas, 2(2), 17-31.

Daft, R. (2005). Teoría y diseño organizacional. $8^{\mathrm{a}}$ edic. México: Thomson.

Domínguez-Aroca, M. (2012). Lucha contra el plagio desde las bibliotecas universitarias. El Profesional de la Información, 21 (5), pp. 498-503. Disponible en: http://eprints.rclis. org/17727/1/Plagio-BU-2012.pdf

Gamboa-Suárez, A. A. (2016). Docencia, investigación y gestión: Reflexiones sobre su papel en la calidad de la educación superior. Revista Perspectivas, 1(1), 81-90.

García Romero, M. (2017). Inventario de artículos de investigación sobre escrituraylectura académica producidos por miembros de la Universidad de Los Andes. Acción Pedagógica, 26, pp. 176-184. Disponible en: http://www.saber.ula. ve/bitstream/handle/123456789/44482/art7. pdf? sequence $=1$ \&isAllowed $=\mathrm{y}$

Guerrero, R. y Zambrano, J. (2012). Las investigaciones sobre escritura académica en la Universidad de Los Andes (Venezuela). Lengua y Habla, 16, pp. 203-224. Disponible en: http://erevistas.saber. ula.ve/index.php/ lenguayhabla/article/view/4151

Giordan, A. y De Vecchi, G. (1995). Los orígenes del saber. De las concepciones personales a los conceptos cientificos. $2^{\mathrm{a}}$ edic. Sevilla: Díada.

González, B. y Salazar, A. (2015). Formación inicial en lectura y escritura en la universidad. De la educación media al desempeño académico en la educación superior. Bogotá: Pontificia Universidad Javeriana. pp. 135-178.

Latorre, A.; del Rincón, D.; y Arnal, J. (2005). Bases metodológicas de la investigación educativa. Barcelona: Ediciones Experiencia.
Lomas, C. y Ruiz, U. (2002). Imitar, plagiar, crear. Textos de Didáctica de la Lengua y de la Literatura, VII (30), pp. 5-7.

Lozano, M.; Hernández, G.; Ortíz, E. y Chacón, L. (2015). Prácticas de escritura. En: González, B. y Salazar, A. (2015). Formación inicial en lectura y escritura en la universidad. De la educación media al desempeño académico en la educación superior. Bogotá: Pontificia Universidad Javeriana. pp. 135-178.

Martín, M. M., Hernández-Suarez, C. A., \& Mendoza-Lizcano, S. M. (2017). Ambientes de aprendizaje basados en herramientas web para el desarrollo de competencias TIC en la docencia. Revista Perspectivas, 2(1), 97-104.

Martins, I. (2012). El pensamiento de profesor sobre la escritura académica y profesional: un estudio de caso de la Escuela de Derecho de la Universidad Central de Venezuela. Legenda, 16 (15), 72-93. Disponible en: http://erevistas. saber.ula.ve/index.php/legenda/article/ view/4263/4050

Morales, O. (2000) Concepciones teóricas sobre lectura y escritura y su aprendizaje: estudio con dos docentes de primera etapa de Educación Básica. Memorias del $5^{\circ}$ Congreso Colombiano y $4^{\circ}$ Latinoamericano de Lectura y Escritura. Mérida: Universidad de Los Andes, Ediciones del Postgrado de Lectura y Escritura.

Moreno, M. y Azcárate, C. (2003). Concepciones $\mathrm{y}$ creencias de los profesores universitarios de matemáticas acerca de la enseñanza de las ecuaciones diferenciales. Enseñanza de las Ciencias, 21 (2), pp. 265-280.

Mostacero, R. (2012). Dificultades de escritura en el discurso académico: análisis crítico de una situación problemática. Legenda, 16 (14), 63 88. Disponible en: http://erevistas.saber.ula.ve/ index.php/legenda/issue/view/290

Pozo, J.A. (2009). Adquirir una concepción compleja del conocimiento: Creencias epistemológicas y concepciones del aprendizaje. En: Pozo, J. A. y Pérez Echeverría, M. (coords.) Psicología 
del aprendizaje universitario: La formación en competencias (pp. 70-85). Madrid: Morata.

Ramírez, F. (2017). La enseñanza de la lectura y la escritura en las comunidades académicas universitarias. Educación Superior y Sociedad, 18 Escritura académica, investigación y desarrollo epistémico, 59-82. Disponible en: http://ess.iesalc.unesco.org.ve/ess3/index.php/ ess/issue/view/39

Red de Lectura y Escritura en la Educación SuperiorAsociación Colombiana de Universidades (REDLEES-ASCUN, 2013). Reflexiones y propuestasparapensarunapolíticainstitucional de desarrollo de la lectura y la escritura en Educación Superior. Documento de trabajo, $4^{\mathrm{a}}$ versión. Disponible en: http://www.ascunredlees.org/wp-content/uploads/2013/07/ Documento-de-pol\%C3\%ADticas-16-dejunio-cuarto-borrador.pdf

Rincón, G. y Gil, J. S. (2010). Las prácticas de lectura y escritura académicas en la Universidad del Valle: tendencias. Lenguaje, 38 (2), 387419. Disponible en: https://media.utp.edu.co/ referencias-bibliograficas/uploads/referencias/ articulo/1057-las-practicas-de-lectura-y-deescritura-academicas-en-la-universidad-delvalle-tendenciaspdf-yhgZt-articulo.pdf

Rojas, G. y Jiménez, H. (2012). La educación superior desde la lectura y la escritura. Amazonia Investiga, 3 (1), pp. 19-35. Disponible en: file://C:/Documents $\% 20$ and $\% 20$ Settings/Administrador/Mis $\% 20$ documentos/Downloads/922-5623-1-PB\%20 (2).pdf

Serrano, S., Duque, Y., y Madrid, A. (2012). Prácticas de escritura académica en la universidad: ¿reproducir o transformar? Educere, 16 (53), 93-108. Disponible en: http://www.redalyc. org/articulo.oa? id=35623538011

Strauss, A. y Corbin, J. (2002). Bases de la investigación cualitativa. Técnicas y procedimientos para desarrollar la teoría fundamentada. Colección Contus. Medellín: Universidad de Antioquía.
Taylor, S. J. y Bogdan, R. Introducción a los métodos cualitativos en investigación. La búsqueda de los significados. Barcelona: Paidós.

Toca, C. y Carrillo, J. (2009). Asuntos teóricos y metodológicos de la cultura organizacional. En: Civilizar, 9 (17), pp. 117-136. Disponible en: http://www.usergioarboleda.edu.co/civilizar/ civilizar\%20\%2017/Civ\%2017.\%20art.\%206. pdf

Universidad de los Andes, Núcleo Universitario Dr. Pedro Rincón Gutiérrez. Coordinación Académica - Coordinación Comisión Curricular (2009). Diseño Curricular Carrera de Educación-Mención Básica Integral. Reestructuración. San Cristóbal, Táchira.

Urbina, S. (2004). Ciberplagio: "construyendo" trabajos universitarios. Ponencia presentada en el Congreso Internacional sobre Educación y Tecnologías de la Información y Comunicación (Edutec, 2004), 17 al 19 de noviembre, Barcelona: Universitat de Barcelona. Disponible en: http://edutec2004.1mi.ub.es/ pdf/159.pdf

Villamizar-Acevedo, G. A., Lozano-León, S. G., \& Sierra-Garavito, E. D. (2017). Creencias sobre las fuentes y formas de acceso al conocimiento generadas en las prácticas pedagógicas desde la perspectiva del estudiante. Revista Perspectivas, 2(1), 18-27.

Wesseler, M. (2004) La confianza importa: Cómo la cultura institucional puede favorecer la gestión de la calidad y el cambio de la educación superior. Unicambio21. Módulo 2. Panamá.

Zambrano, J. y Guerrero, R. (2015). Escribir en la Universidad: Una Revisión de las Investigaciones de la Universidad de Los Andes, Venezuela. Íkala, 20 (3), 385- 398. Disponible en: http://www.redalyc.org/ pdf/2550/255042795007.pdf 